\title{
Comment on "Functional inequalities associated with Jordan-von Neumann type additive functional equations"
}

\author{
Choonkil Park ${ }^{1}$ and Jung Rye Lee $2^{2^{*}}$
}

* Correspondence: jrlee@daejin.ac. $\mathrm{kr}$

${ }^{2}$ Department of Mathematics, Daejin University, Kyeonggi 487711, Korea

Full list of author information is available at the end of the article

\section{Abstract}

Park et al. proved the Hyers-Ulam stability of some additive functional inequalities. There is a fatal error in the proof of Theorem 3.1. We revise the statements of the main theorems and prove the revised theorems.

2010 Mathematics Subject Classification: Primary 39B62; 39B72; 39852.

Keywords: Jordan-von Neumann functional equation, Hyers-Ulam stability, functional inequality

\section{Introduction and preliminaries}

Ulam [1] gave a talk before the Mathematics Club of the University of Wisconsin in which he discussed a number of unsolved problems. Among these was the following question concerning the stability of homomorphisms.

We are given a group $G$ and a metric group $G$ ' with metric $\rho(\cdot, \cdot)$. Given $\epsilon>0$, does there exist a $\delta>0$ such that if $f: G \rightarrow G$ ' satisfies $\rho(f(x y), f(x) f(y))<\delta$ for all $x, y \in G$, then a homomorphism $h: G \rightarrow G$ ' exists with $\rho(f(x), h(x))<\epsilon$ for all $x \in G$ ?

Hyers [2] considered the case of approximately additive mappings $f: E \rightarrow E$, where $E$ and $E^{\prime}$ are Banach spaces and $f$ satisfies Hyers' inequality

$$
\|f(x+y)-f(x)-f(y)\| \leq \varepsilon
$$

for all $x, y \in E$. It was shown that the limit

$$
L(x)=\lim _{n \rightarrow \infty} \frac{f\left(2^{n} x\right)}{2^{n}}
$$

exists for all $x \in E$ and that $L: E \rightarrow E^{\prime}$ is the unique additive mapping satisfying

$$
\|f(x)-L(x)\| \leq \varepsilon .
$$

Rassias [3] provided a generalization of Hyers' Theorem which allows the Cauchy difference to be unbounded.

Theorem 1.1. (Rassias). Let $f: E \rightarrow E$ ' be a mapping from a normed vector space $E$ into a Banach space E' subject to the inequality

$$
\|f(x+y)-f(x)-f(y)\| \leq \varepsilon\left(\|x\|^{p}+\|y\|^{p}\right)
$$

(c) 2012 Park and Lee; licensee Springer. This is an open access article distributed under the terms of the Creative Commons Attribution License (http://creativecommons.org/licenses/by/2.0), which permits unrestricted use, distribution, and reproduction in any medium, provided the original work is properly cited. 
for all $x, y \in E$, where $\epsilon$ and $p$ are constants with $\epsilon>0$ and $p<1$. Then the limit

$$
L(x)=\lim _{n \rightarrow \infty} \frac{f\left(2^{n} x\right)}{2^{n}}
$$

exists for all $x \in E$ and $L: E \rightarrow E^{\prime}$ is the unique additive mapping which satisfies

$$
\|f(x)-L(x)\| \leq \frac{2 \varepsilon}{2-2^{p}}\|x\|^{p}
$$

for all $x \in E$. If $p<0$ then inequality (1.1) holds for $x, y \neq 0$ and (1.2) for $x \neq 0$.

Rassias [4] during the 27th International Symposium on Functional Equations asked the question whether such a theorem can also be proved for $p \geq 1$. Gajda [5] following the same approach as in Rassias [3] gave an affirmative solution to this question for $p$ $>1$. It was shown by Gajda [5], as well as by Rassias and Šemrl [6] that one cannot prove a Rassias' type theorem when $p=1$ (cf. the books of Czerwik [7] and Hyers et al. [8]).

Rassias [9] followed the innovative approach of Rassias' theorem [3] in which he replaced the factor $\|x\|^{p}+\|y\|^{p}$ by $\|x\|^{p} \cdot\|y\|^{q}$ for $p, q \in \mathbb{R}$ with $p+q \neq 1$. Găvruta [10] provided a further generalization of Rassias' theorem. During the last two decades a number of papers and research monographs have been published on various generalizations and applications of the Hyers-Ulam stability to a number of functional equations and mappings (see [11-13]).

Throughout this article, let $G$ be a 2-divisible abelian group. Assume that $X$ is a normed space with norm $\|\cdot\|_{X}$ and that $Y$ is a Banach space with norm $\|\cdot\|_{Y}$.

Gilányi [14] showed that if $f$ satisfies the functional inequality

$$
\left\|2 f(x)+2 f(y)-f\left(x y^{-1}\right)\right\| \leq\|f(x y)\|
$$

then $f$ satisfies the Jordan-von Neumann functional equation

$$
2 f(x)+2 f(y)=f(x y)+f\left(x y^{-1}\right) .
$$

See also [15]. Gilányi [16] and Fechner [17] proved the Hyers-Ulam stability of the functional inequality (1.3).

Park et al. [18] proved the Hyers-Ulam stability of the following functional inequalities

$$
\begin{aligned}
& \|f(x)+f(y)+f(z)\| \leq\left\|2 f\left(\frac{x+y+z}{2}\right)\right\|, \\
& \|f(x)+f(y)+f(z)\| \leq\|f(x+y+z)\|, \\
& \|f(x)+f(y)+2 f(z)\| \leq\left\|2 f\left(\frac{x+y}{2}+z\right)\right\| .
\end{aligned}
$$

But there is an error in the 8th line on the 6th page in the proof of [18, Theorem 3.1]. We revise the statements of the main theorems and prove the revised theorems.

In Section 2, we prove the Hyers-Ulam stability of the functional inequality (1.4). In Section 3, we prove the Hyers-Ulam stability of the functional inequality (1.5). In Section 4, we prove the Hyers-Ulam stability of the functional inequality (1.6). 


\section{Stability of a functional inequality associated with a 3-variable Jensen additive functional equation}

Proposition 2.1. [18, Proposition 2.1] Let $f: G \rightarrow Y$ be a mapping such that

$$
\|f(x)+f(y)+f(z)\|_{Y} \leq\left\|2 f\left(\frac{x+y+z}{2}\right)\right\|_{Y}
$$

for all $x, y, z \in G$. Then $f$ is Cauchy additive.

We prove the Hyers-Ulam stability of a functional inequality associated with a Jordan-von Neumann type 3-variable Jensen additive functional equation.

Theorem 2.2. Let $r>1$ and $\theta$ be nonnegative real numbers, and let $f: X \rightarrow Y$ be an odd mapping such that

$$
\|f(x)+f(y)+f(z)\|_{Y} \leq\left\|2 f\left(\frac{x+y+z}{2}\right)\right\|_{Y}+\theta\left(\|x\|_{X}^{r}+\|y\|_{X}^{r}+\|z\|_{X}^{r}\right)
$$

for all $x, y, z \in X$. Then there exists a unique Cauchy additive mapping $h: X \rightarrow Y$ such that

$$
\|f(x)-h(x)\|_{Y} \leq \frac{2^{r}+2}{2^{r}-2} \theta\|x\|_{X}^{r}
$$

for all $x \in X$.

Proof. Letting $y=x$ and $z=-2 x$ in (2.1), we get

$$
\|2 f(x)-f(2 x)\|_{Y}=\|2 f(x)+f(-2 x)\|_{Y} \leq\left(2+2^{r}\right) \theta\|x\|_{X}^{r}
$$

for all $x \in X$. So

$$
\left\|f(x)-2 f\left(\frac{x}{2}\right)\right\|_{Y} \leq \frac{2+2^{r}}{2^{r}} \theta\|x\|_{X}^{r}
$$

for all $x \in X$. Hence

$$
\begin{aligned}
\left\|2^{l} f\left(\frac{x}{2^{l}}\right)-2^{m} f\left(\frac{x}{2^{m}}\right)\right\|_{Y} & \leq \sum_{j=l}^{m-1}\left\|2^{j} f\left(\frac{x}{2^{j}}\right)-2^{j+1} f\left(\frac{x}{2^{j+1}}\right)\right\|_{Y} \\
& \leq \frac{2+2^{r}}{2^{r}} \sum_{j=l}^{m-1} \frac{2^{j}}{2^{r j}} \theta\|x\|_{X}^{r}
\end{aligned}
$$

for all nonnegative integers $m$ and $l$ with $m>l$ and all $x \in X$.

It follows from (2.4) that the sequence $\left\{2^{n} f\left(\frac{x}{2^{n}}\right)\right\}$ is a Cauchy sequence for all $x \in X$. Since $Y$ is complete, the sequence $\left\{2^{n} f\left(\frac{x}{2^{n}}\right)\right\}$ converges. So one can define the mapping $h: X \rightarrow Y$ by

$$
h(x):=\lim _{n \rightarrow \infty} 2^{n} f\left(\frac{x}{2^{n}}\right)
$$

for all $x \in X$. Moreover, letting $l=0$ and passing the limit $m \rightarrow \infty$ in (2.4), we get (2.2). 
It follows from (2.1) that

$$
\begin{aligned}
\| h(x)+h(y) & +h(z)\left\|_{Y}=\lim _{n \rightarrow \infty} 2^{n}\right\| f\left(\frac{x}{2^{n}}\right)+f\left(\frac{y}{2^{n}}\right)+f\left(\frac{z}{2^{n}}\right) \|_{Y} \\
& \leq \lim _{n \rightarrow \infty} 2^{n}\left\|2 f\left(\frac{x+y+z}{2^{n+1}}\right)\right\|_{Y}+\lim _{n \rightarrow \infty} \frac{2^{n} \theta}{2^{n r}}\left(\|x\|_{X}^{r}+\|y\|_{X}^{r}+\|z\|_{X}^{r}\right) \\
& =\left\|2 h\left(\frac{x+y+z}{2}\right)\right\|_{Y}
\end{aligned}
$$

for all $x, y, z \in X$. So

$$
\|h(x)+h(y)+h(z)\|_{Y} \leq\left\|2 h\left(\frac{x+y+z}{2}\right)\right\|_{Y}
$$

for all $x, y, z \in X$. By Proposition 2.1, the mapping $h: X \rightarrow Y$ is Cauchy additive.

Now, let $T: X \rightarrow Y$ be another Cauchy additive mapping satisfying (2.2). Then we have

$$
\begin{aligned}
\|h(x)-T(x)\|_{Y} & =2^{n}\left\|h\left(\frac{x}{2^{n}}\right)-T\left(\frac{x}{2^{n}}\right)\right\|_{Y} \\
& \leq 2^{n}\left(\left\|h\left(\frac{x}{2^{n}}\right)-f\left(\frac{x}{2^{n}}\right)\right\|_{Y}+\left\|T\left(\frac{x}{2^{n}}\right)-f\left(\frac{x}{2^{n}}\right)\right\|_{Y}\right) \\
& \leq \frac{2\left(2^{r}+2\right) 2^{n}}{\left(2^{r}-2\right) 2^{n r}} \theta\|x\|_{X}^{r},
\end{aligned}
$$

which tends to zero as $n \rightarrow \infty$ for all $x \in X$. So we can conclude that $h(x)=T(x)$ for all $x \in X$. This proves the uniqueness of $h$. Thus the mapping $h: X \rightarrow Y$ is a unique Cauchy additive mapping satisfying (2.2).

Theorem 2.3. Let $r<1$ and $\theta$ be positive real numbers, and let $f: X \rightarrow Y$ be an odd mapping satisfying (2.1). Then there exists a unique Cauchy additive mapping $h: X \rightarrow$ $Y$ such that

$$
\|f(x)-h(x)\|_{Y} \leq \frac{2+2^{r}}{2-2^{r}} \theta\|x\|_{X}^{r}
$$

for all $x \in X$.

Proof. It follows from (2.3) that

$$
\left\|f(x)-\frac{1}{2} f(2 x)\right\|_{Y} \leq \frac{2+2^{r}}{2} \theta\|x\|_{X}^{r}
$$

for all $x \in X$. Hence

$$
\begin{aligned}
\left\|\frac{1}{2^{l}} f\left(2^{l} x\right)-\frac{1}{2^{m}} f\left(2^{m} x\right)\right\|_{Y} & \leq \sum_{j=l}^{m-1}\left\|\frac{1}{2^{j}} f\left(2^{j} x\right)-\frac{1}{2^{j+1}} f\left(2^{j+1} x\right)\right\|_{Y} \\
& \leq \frac{2+2^{r}}{2} \sum_{j=l}^{m-1} \frac{2^{r j}}{2^{j}} \theta\|x\|_{X}^{r}
\end{aligned}
$$

for all nonnegative integers $m$ and $l$ with $m>l$ and all $x \in X$.

It follows from (2.6) that the sequence $\left\{\frac{1}{2^{n}} f\left(2^{n} x\right)\right\}$ is a Cauchy sequence for all $x \in$ $X$. Since $Y$ is complete, the sequence $\left\{\frac{1}{2^{n}} f\left(2^{n} x\right)\right\}$ converges. So one can define the mapping $h: X \rightarrow Y$ by 


$$
h(x):=\lim _{n \rightarrow \infty} \frac{1}{2^{n}} f\left(2^{n} x\right)
$$

for all $x \in X$. Moreover, letting $l=0$ and passing the limit $m \rightarrow \infty$ in (2.6), we get (2.5).

The rest of the proof is similar to the proof of Theorem 2.2.

Theorem 2.4. Let $r>\frac{1}{3}$ and $\theta$ be nonnegative real numbers, and let $f: X \rightarrow Y$ be an odd mapping such that

$$
\|f(x)+f(y)+f(z)\|_{Y} \leq\left\|2 f\left(\frac{x+y+z}{2}\right)\right\|_{Y}+\theta \cdot\|x\|_{X}^{r} \cdot\|y\|_{X}^{r} \cdot\|z\|_{X}^{r}
$$

for all $x, y, z \in X$. Then there exists a unique Cauchy additive mapping $h: X \rightarrow Y$ such that

$$
\|f(x)-h(x)\|_{Y} \leq \frac{2^{r} \theta}{8^{r}-2}\|x\|_{X}^{3 r}
$$

for all $x \in X$.

Proof. Letting $y=x$ and $z=-2 x$ in (2.7), we get

$$
\|2 f(x)-f(2 x)\|_{Y}=\|2 f(x)+f(-2 x)\|_{Y} \leq 2^{r} \theta\|x\|_{X}^{3 r}
$$

for all $x \in X$. So

$$
\left\|f(x)-2 f\left(\frac{x}{2}\right)\right\|_{Y} \leq \frac{2^{r}}{8^{r}} \theta\|x\|_{X}^{3 r}
$$

for all $x \in X$. Hence

$$
\begin{aligned}
\left\|2^{l} f\left(\frac{x}{2^{l}}\right)-2^{m} f\left(\frac{x}{2^{m}}\right)\right\|_{Y} & \leq \sum_{j=l}^{m-1}\left\|2^{j} f\left(\frac{x}{2^{j}}\right)-2^{j+1} f\left(\frac{x}{2^{j+1}}\right)\right\|_{Y} \\
& \leq \frac{2^{r}}{8^{r}} \sum_{j=l}^{m-1} \frac{2^{j}}{8^{r j}} \theta\|x\|_{X}^{3 r}
\end{aligned}
$$

for all nonnegative integers $m$ and $l$ with $m>l$ and all $x \in X$.

It follows from (2.10) that the sequence $\left\{2^{n} f\left(\frac{x}{2^{n}}\right)\right\}$ is a Cauchy sequence for all $x \in$ $X$. Since $Y$ is complete, the sequence $\left\{2^{n} f\left(\frac{x}{2^{n}}\right)\right\}$ converges. So one can define the mapping $h: X \rightarrow Y$ by

$$
h(x):=\lim _{n \rightarrow \infty} 2^{n} f\left(\frac{x}{2^{n}}\right)
$$

for all $x \in X$. Moreover, letting $l=0$ and passing the limit $m \rightarrow \infty$ in (2.10), we get (2.8).

The rest of the proof is similar to the proof of Theorem 2.2.

Theorem 2.5. Let $r<\frac{1}{3}$ and $\theta$ be positive real numbers, and let $f: X \rightarrow Y$ be an odd mapping satisfying (2.7). Then there exists a unique Cauchy additive mapping h:X $\rightarrow Y$ such that 


$$
\|f(x)-h(x)\|_{Y} \leq \frac{2^{r} \theta}{2-8^{r}}\|x\|_{X}^{3 r}
$$

for all $x \in X$.

Proof. It follows from (2.9) that

$$
\left\|f(x)-\frac{1}{2} f(2 x)\right\|_{Y} \leq \frac{2^{r}}{2} \theta\|x\|_{X}^{3 r}
$$

for all $x \in X$. Hence

$$
\begin{aligned}
\left\|\frac{1}{2^{l}} f\left(2^{l} x\right)-\frac{1}{2^{m}} f\left(2^{m} x\right)\right\|_{Y} & \leq \sum_{j=l}^{m-1}\left\|\frac{1}{2^{j}} f\left(2^{j} x\right)-\frac{1}{2^{j+1}} f\left(2^{j+1} x\right)\right\|_{Y} \\
& \leq \frac{2^{r}}{2} \sum_{j=l}^{m-1} \frac{8^{r j}}{2^{j}} \theta\|x\|_{X}^{r}
\end{aligned}
$$

for all nonnegative integers $m$ and $l$ with $m>l$ and all $x \in X$.

It follows from (2.12) that the sequence $\left\{\frac{1}{2^{n}} f\left(2^{n} x\right)\right\}$ is a Cauchy sequence for all $x \in$ $X$. Since $Y$ is complete, the sequence $\left\{\frac{1}{2^{n}} f\left(2^{n} x\right)\right\}$ converges. So one can define the mapping $h: X \rightarrow Y$ by

$$
h(x):=\lim _{n \rightarrow \infty} \frac{1}{2^{n}} f\left(2^{n} x\right)
$$

for all $x \in X$. Moreover, letting $l=0$ and passing the limit $m \rightarrow \infty$ in (2.12), we get (2.11).

The rest of the proof is similar to the proof of Theorem 2.2.

\section{Stability of a functional inequality associated with a 3-variable Cauchy additive functional equation}

Proposition 3.1. [18, Proposition 2.2] Let $f: G \rightarrow Y$ be a mapping such that

$$
\|f(x)+f(y)+f(z)\|_{Y} \leq\|f(x+y+z)\|_{Y}
$$

for all $x, y, z \in G$. Then $f$ is Cauchy additive.

We prove the Hyers-Ulam stability of a functional inequality associated with a Jordan-von Neumann type 3-variable Cauchy additive functional equation.

Theorem 3.2. Let $r>1$ and $\theta$ be nonnegative real numbers, and let $f: X \rightarrow Y$ be an odd mapping such that

$$
\|f(x)+f(y)+f(z)\|_{Y} \leq\|f(x+y+z)\|_{Y}+\theta\left(\|x\|_{X}^{r}+\|y\|_{X}^{r}+\|z\|_{X}^{r}\right)
$$

for all $x, y, z \in X$. Then there exists a unique Cauchy additive mapping $h: X \rightarrow Y$ such that

$$
\|f(x)-h(x)\|_{Y} \leq \frac{2^{r}+2}{2^{r}-2} \theta\|x\|_{X}^{r}
$$

for all $x \in X$. 
Proof. Letting $y=x$ and $z=-2 x$ in (3.1), we get

$$
\|2 f(x)-f(2 x)\|_{Y}=\|2 f(x)+f(-2 x)\|_{Y} \leq\left(2+2^{r}\right) \theta\|x\|_{X}^{r}
$$

for all $x \in X$.

The rest of the proof is the same as in the proof of Theorem 2.2.

Theorem 3.3. Let $r<1$ and $\theta$ be positive real numbers, and let $f: X \rightarrow Y$ be an odd mapping satisfying (3.1). Then there exists a unique Cauchy additive mapping $h: X \rightarrow Y$ such that

$$
\|f(x)-h(x)\|_{Y} \leq \frac{2+2^{r}}{2-2^{r}} \theta\|x\|_{X}^{r}
$$

for all $x \in X$.

Proof. It follows from (3.2) that

$$
\left\|f(x)-\frac{1}{2} f(2 x)\right\|_{Y} \leq \frac{2+2^{r}}{2} \theta\|x\|_{X}^{r}
$$

for all $x \in X$.

The rest of the proof is the same as in the proofs of Theorems 2.2 and 2.3.

Theorem 3.4. Let $r>\frac{1}{3}$ and $\theta$ be nonnegative real numbers, and let $f: X \rightarrow Y$ be an odd mapping such that

$$
\|f(x)+f(y)+f(z)\|_{Y} \leq\|f(x+y+z)\|_{Y}+\theta \cdot\|x\|_{X}^{r} \cdot\|y\|_{X}^{r} \cdot\|z\|_{X}^{r}
$$

for all $x, y, z \in X$. Then there exists a unique Cauchy additive mapping $h: X \rightarrow Y$ such that

$$
\|f(x)-h(x)\|_{Y} \leq \frac{2^{r} \theta}{8^{r}-2}\|x\|_{X}^{3 r}
$$

for all $x \in X$.

Proof Letting $y=x$ and $z=-2 x$ in (3.3), we get

$$
\|2 f(x)-f(2 x)\|_{Y}=\|2 f(x)+f(-2 x)\|_{Y} \leq 2^{r} \theta\|x\|_{X}^{3 r}
$$

for all $x \in X$.

The rest of the proof is the same as in the proofs of Theorems 2.2 and 2.4.

Theorem 3.5. Let $r<\frac{1}{3}$ and $\theta$ be positive real numbers, and let $f: X \rightarrow Y$ be an odd mapping satisfying (3.3). Then there exists a unique Cauchy additive mapping $h: X \rightarrow$ $Y$ such that

$$
\|f(x)-h(x)\|_{Y} \leq \frac{2^{r} \theta}{2-8^{r}}\|x\|_{X}^{3 r}
$$

for all $x \in X$.

Proof. It follows from (3.4) that

$$
\left\|f(x)-\frac{1}{2} f(2 x)\right\|_{Y} \leq \frac{2^{r}}{2} \theta\|x\|_{X}^{3 r}
$$

for all $x \in X$. 
The rest of the proof is the same as in the proofs of Theorems 2.2 and 2.5.

\section{Stability of a functional inequality associated with the Cauchy-Jensen functional equation}

Proposition 4.1. [18, Proposition 2.3] Let $f: G \rightarrow Y$ be a mapping such that

$$
\|f(x)+f(y)+2 f(z)\|_{Y} \leq\left\|2 f\left(\frac{x+y}{2}+z\right)\right\|_{Y}
$$

for all $x, y, z \in G$. Then $f$ is Cauchy additive.

We prove the Hyers-Ulam stability of a functional inequality associated with a Jordan-von Neumann type Cauchy-Jensen functional equation.

Theorem 4.2. Let $r>1$ and $\theta$ be nonnegative real numbers, and let $f: X \rightarrow Y$ be an odd mapping such that

$$
\|f(x)+f(y)+2 f(z)\|_{Y} \leq\left\|2 f\left(\frac{x+y}{2}+z\right)\right\|_{Y}+\theta\left(\|x\|_{X}^{r}+\|y\|_{X}^{r}+\|z\|_{X}^{r}\right)
$$

for all $x, y, z \in X$. Then there exists a unique Cauchy additive mapping $h: X \rightarrow Y$ such that

$$
\|f(x)-h(x)\|_{Y} \leq \frac{2^{r}+1}{2^{r}-2} \theta\|x\|_{X}^{r}
$$

for all $x \in X$.

Proof. Replacing $x$ by $2 x$ and letting $y=0$ and $z=-x$ in (4.1), we get

$$
\|f(2 x)-2 f(x)\|_{Y}=\|f(2 x)+2 f(-x)\|_{Y} \leq\left(1+2^{r}\right) \theta\|x\|_{X}^{r}
$$

for all $x \in X$. So

$$
\left\|f(x)-2 f\left(\frac{x}{2}\right)\right\|_{Y} \leq \frac{1+2^{r}}{2^{r}} \theta\|x\|_{X}^{r}
$$

for all $x \in X$.

The rest of the proof is similar to the proof of Theorem 2.2.

Theorem 4.3. Let $r<1$ and $\theta$ be positive real numbers, and let $f: X \rightarrow Y$ be an odd mapping satisfying (4.1). Then there exists a unique Cauchy additive mapping $h: X \rightarrow$ $Y$ such that

$$
\|f(x)-h(x)\|_{Y} \leq \frac{1+2^{r}}{2-2^{r}} \theta\|x\|_{X}^{r}
$$

for all $x \in X$.

Proof. It follows from (4.2) that

$$
\left\|f(x)-\frac{1}{2} f(2 x)\right\|_{Y} \leq \frac{1+2^{r}}{2} \theta\|x\|_{X}^{r}
$$

for all $x \in X$.

The rest of the proof is similar to the proofs of Theorems 2.2 and 2.3 .

\section{Author details}

${ }^{1}$ Department of Mathematics, Hanyang University, Seoul 133-791, Korea ²Department of Mathematics, Daejin

University, Kyeonggi 487-711, Korea 


\section{Authors' contributions}

All authors conceived of the study, participated in its design and coordination, drafted the manuscript, participated in the sequence alignment, and read and approved the final manuscript.

\section{Competing interests}

The authors declare that they have no competing interests.

Received: 8 November 2011 Accepted: 28 February 2012 Published: 28 February 2012

\section{References}

1. Ulam, SM: A Collection of the Mathematical Problems. Interscience Publ, New York (1960)

2. Hyers, DH: On the stability of the linear functional equation. Proc Natl Acad Sci USA. 27, 222-224 (1941). doi:10.1073/ pnas.27.4.222

3. Rassias, ThM: On the stability of the linear mapping in Banach spaces. Proc Am Math Soc. 72, 297-300 (1978). doi:10.1090/S0002-9939-1978-0507327-1

4. Rassias, ThM: Problem 16; 2 report of the $27^{\text {th }}$ international symposium on functional equations. Aequationes Math 39 , 292-293 (1990). 309

5. Gajda, Z: On stability of additive mappings. Int J Math Math Sci. 14, 431-434 (1991). doi:10.1155/S016117129100056X

6. Rassias, ThM, Šemrl, P: On the behaviour of mappings which do not satisfy Hyers-Ulam stability. Proc Am Math Soc. 114, 989-993 (1992). doi:10.1090/50002-9939-1992-1059634-1

7. Czerwik, P: Functional Equations and Inequalities in Several Variables. World Scientific Publishing Company, New Jersey, Hong Kong, Singapore, London (2002)

8. Hyers, DH, Isac, G, Rassias, ThM: Stability of Functional Equations in Several Variables. Birkhäuser, Basel (1998)

9. Rassias, JM: On approximation of approximately linear mappings by linear mappings. J Funct Anal. 46, 126-130 (1982). doi:10.1016/0022-1236(82)90048-9

10. Găvruta, P: A generalization of the Hyers-Ulam-Rassias stability of approximately additive mappings. J Math Anal Appl. 184, 431-436 (1994). doi:10.1006/jmaa.1994.1211

11. Jun, K, Lee, Y: A generalization of the Hyers-Ulam-Rassias stability of the Pexiderized quadratic equations. J Math Anal Appl. 297, 70-86 (2004). doi:10.1016/j.jmaa.2004.04.009

12. Jung, S: Hyers-Ulam-Rassias Stability of Functional Equations in Mathematical Analysis. Hadronic Press Inc., Palm Harbor, Florida (2001)

13. Park, C: Homomorphisms between Poisson JC-algebras. Bull Braz Math Soc. 36, 79-97 (2005). doi:10.1007/s00574-0050029-z

14. Gilányi, A: Eine zur parallelogrammgleichung äquivalente ungleichung. Aequationes Math. 62, 303-309 (2001). doi:10.1007/PL00000156

15. Rätz, J: On inequalities associated with the Jordan-von Neumann functional equation. Aequa-tiones Math. 66, 191-200 (2003). doi:10.1007/s00010-003-2684-8

16. Gilányi, A: On a problem by K. Nikodem Math Inequal Appl. 5, 707-710 (2002)

17. Fechner, W: Stability of a functional inequalities associated with the Jordan-von Neumann functional equation. Aequationes Math. 71, 149-161 (2006). doi:10.1007/s00010-005-2775-9

18. Park, C, Cho, Y, Han, M: Functional inequalities associated with Jordan-von Neumann-type additive functional equations. J Inequal Appl 2007 (2007). Article ID 41820, 13

doi:10.1186/1029-242X-2012-47

Cite this article as: Park and Lee: Comment on "Functional inequalities associated with Jordan-von Neumann type additive functional equations". Journal of Inequalities and Applications 2012 2012:47.

\section{Submit your manuscript to a SpringerOpen ${ }^{\odot}$ journal and benefit from:}

Convenient online submission

- Rigorous peer review

- Immediate publication on acceptance

- Open access: articles freely available online

- High visibility within the field

- Retaining the copyright to your article

Submit your next manuscript at $\boldsymbol{s p r i n g e r o p e n . c o m ~}$ 